RBMC

ISSN 2447-9071

doi https://doi.org/10.36414/rbmc.v7i19.103

Contato para correspondência: Hermínio Maurício da Rocha Sobrinho

E-mail:

herminio.sobrinho@gmail.com

Conflito de interesse: Não

Financiamento: Recursos próprios

Recebido: 12/10/2021

Aprovado: 07/12/2021

\section{A influência da musicoterapia no tratamento auxiliar da doença de Parkinson: uma revisão da literatura}

\author{
The influence of musicotherapy in Parkinson's disease auxiliary \\ treatment: a review
}

Marcelo Borges Figueira da Mota', Renata Castro Fagundes Bomfim', Tereza Raquel Alcântara-Silva², Delson José da Silva², Hermínio Maurício da Rocha Sobrinho ${ }^{1,3}$

\author{
'Pontifícia Universidade Católica de Goiás - PUC Goiás \\ ${ }^{2}$ Universidade Federal de Goiás - UFG \\ ${ }^{3}$ Universidade Estadual de Goiás - UEG
}

\section{Resumo}

Adoença de Parkinson (DP)écaracterizada, principalmente, por uma disfunção do sistema de gânglios basais, produzindo sintomas motores e não motores. O interesse pelo estudo da musicoterapia no tratamento auxiliar de pacientes com DP baseia-se em evidências científicas que comprovam que a músicaécapaz demelhorar a marchaeo comportamento dos pacientes. Tem porobjetivo descreveros principais benefícios, efeitos e a influência da musicoterapia no tratamento auxiliardaDP, enfatizando a sua eficácia no parâmetro motor dos pacientes. Trata-se de uma revisão integrativa da literatura, com seleção dos estudos nas bases de dados Portal de Periódicos da Capes, PubMed, Scielo e SCOPUS, pormeio dos Descritores em Ciências da Saúde (DeCS), nos idiomas português e inglês, no período de 2015 a 2021. Demaneira geral, a terapêutica resultou na melhora do equilíbrio postural, bem comono aumento do comprimento do passo do paciente e no aumento da velocidade da marcha, o que levou a uma maior independência funcional, acarretando desfechos positivos na progressão da doença. $A$ musicoterapia tem se tornado uma ferramenta com grande potencial para o tratamento auxiliar da doença de Parkinson. Esse instrumento tem proporcionado melhor qualidade de vida aos pacientes, aliviando os sintomas motores e não motores ao estimular a produção dopaminérgica.

Palavras-Chave: Doença de Parkinson; Terapias complementares; Musicoterapia.

\begin{abstract}
Parkinson's disease (PD) is characterized mainly by a dysfunction of the basal ganglia system, producing motor and non-motor symptoms. The interest in studying music therapy in the auxiliary treatment of PD patients is based on scientific evidence that proves that music is capable of improving the gait and behavior of patients. The aim is to describe the main benefits, effects, and influence of music therapy in the auxiliary treatment of PD, emphasizing its effectiveness in the motor parameters of patients. Integrative review of the scientific literature worldwide, with selection of studies in the databases Portal of journals of Capes, Pubmed, Scielo and SCOPUS, through health descriptors, in Portuguese and English, in the period from 2015 to 2021. In general, the therapy resulted in an improvement in postural balance, as well as an increase in the patient's stride length and an increase in gait speed, which led to greaterfunctional independence, leading to positive outcomes in the progression of the disease. Music therapy has become a tool with great potential for the auxiliary treatment of Parkinson's disease. This instrument has provided better quality of life to patients, relieving motor and non-motor symptoms by stimulating dopaminergic production.
\end{abstract}

Keywords: Parkinson; Alternative therapies; Music therapy. 


\section{Introdução}

A doença de Parkinson (DP) é uma doença degenerativa do sistema nervoso central ${ }^{1}$, que resulta da morte progressiva de neurônios da substância negra e do lócus ceruleus ${ }^{2}$, ocasionando diminuição da produção dopaminérgica, levando a alterações motoras (bradicinesia, acinesia, tremor de repouso, rigidez muscular e instabilidade postural) e não motoras (destacam-se a depressão, os distúrbios autonômicos, cognitivos e a demência) $)^{1,3,4}$.

A DP é a segunda doença neurodegenerativa mais frequente em todo o mundo ${ }^{5}$, atinge ambos os sexos, com prevalência de homens em relação a mulheres ${ }^{6}$. Espera-se que até 2030, o número de indivíduos que viverão com a doença chegará a 9 milhões ${ }^{7}$. O tratamento da DP abrange o aspecto cirúrgico (estimulação cerebral profunda), o farmacológico e o não farmacológico ${ }^{2}$. A levodopa é o medicamento de maior eficácia dentro da perspectiva farmacológica para o controle sintomático ${ }^{8}$. Terapias alternativas, como: o yoga, fisioterapia, grupos de apoio, dança, exercícios físicos e a musicoterapia são alternativas terapêuticas não farmacológicas que produzem efeitos capazes de aliviar sintomas de doenças neurodegenerativas ${ }^{2}$.

A musicoterapia consiste em um agrupamento de técnicas baseadas na música e seus elementos (som, ritmo, melodia e harmonia), que são utilizadas no tratamento de distúrbios somáticos, psíquicos ou psicossomáticos. Pode ser realizada em grupo ou individual, além de ser categorizada em abordagens ativa ou receptiva. ${ }^{9}$ Essa ferramenta tem se tornado, ao longo dos anos, um potencial terapêutico na área da saúde, principalmente de doenças que acometem a capacidade física, cognitiva e emocional dos indivíduos, como a doença de Parkinson. ${ }^{10,11}$

O interesse pelo estudo da musicoterapia no tratamento auxiliar de pacientes com DP baseia-se em evidências científicas que comprovam que a música é capaz de melhorar a marcha e o comportamento dos doentes. ${ }^{12} \mathrm{O}$ método da estimulação auditiva rítmica (EAR) consiste em um treinamento no qual a marcha dos pacientes é guiada por um estímulo rítmico auditivo. ${ }^{8}$ Esse estímulo é capaz de atuar como modulador da atividade/função cerebral colaborando para minimizar sintomas da doença. ${ }^{6}$

A musicoterapia, no decorrer dos últimos anos, vem se apresentando como um campo de pesquisa altamente promissor para a área da saúde, em suas inúmeras teorias, tem orientado práticas com resultados promissores no tratamento de patologias que afetam a capacidade física, cognitiva ou subjetiva das pessoas, como é o caso de alguns distúrbios neurodegenerativos, como a doença de Parkinson. ${ }^{6,8,12}$
Diante do exposto, o objetivo geral do presente trabalho foi descrever os principais benefícios, efeitos e a influência da musicoterapia no tratamento auxiliar da doença de Parkinson (DP), enfatizando a sua eficácia no parâmetro motor dos pacientes.

\section{Métodos}

Trata-se de uma revisão integrativa da literatura científica, que se configura como a busca por unir o conhecimento atual sobre uma temática específica, de forma a incluir uma variedade mais ampla de estudos, que outras revisões não permitem, abrangendo estudos experimentais e não-experimentais no levantamento de dados. Visa auxiliar na visão crítica do que está sendo publicado cientificamente, apresentando assim, uma visão mais ampla dos dados científicos publicados. ${ }^{27}$ Para a busca dos estudos foram utilizadas as bases de dados eletrônicas: Portal de Periódicos da Capes, PubMed/ MedLine (US National Library of Medicine National Institutes of Health), Scientific Electronic Library Online (SciELO) e SCOPUS Elsevier, utilizando-se os seguintes Descritores em Ciências da Saúde (DeCS) com combinações múltiplas por meio dos operadores boleanos "AND" "eR" entre os termos: doença de Parkinson, musicoterapia, música, neuromusicoterapia e seus correlatos em inglês.

Os artigos foram selecionados segundo os critérios do Preferred Reporting Items for Systematic Reviews and Meta-Analyses $(P R I S M A)^{28}$. A partir do objetivo geral do estudo seguiu-se uma seleção sistematizada em quatro etapas, sendo elas: Identificação, Seleção, Elegibilidade e Inclusão. A busca foi realizada de forma independente por dois pesquisadores e foram confrontados os resultados para se chegar a amostra final.

Foram incluídos artigos publicados no período de 2015 a 2021, nos idiomas português e inglês; que abordem o tema da musicoterapia com sessões realizadas em grupo ou individual; pacientes participantes homens e/ou mulheres de qualquer faixa etária, totalizando 147 artigos. Foram excluídos artigos duplicados, artigos de revisão, trabalhos apresentados em eventos, capítulos de livros e artigos fora dos objetivos propostos, totalizando 121 exclusões. O procedimento metodológico desse estudo é descrito no fluxograma que se segue (Figura 1). Ao final da pesquisa, foram selecionados e analisados 26 artigos, de acordo com os critérios de inclusão estabelecidos neste estudo. 


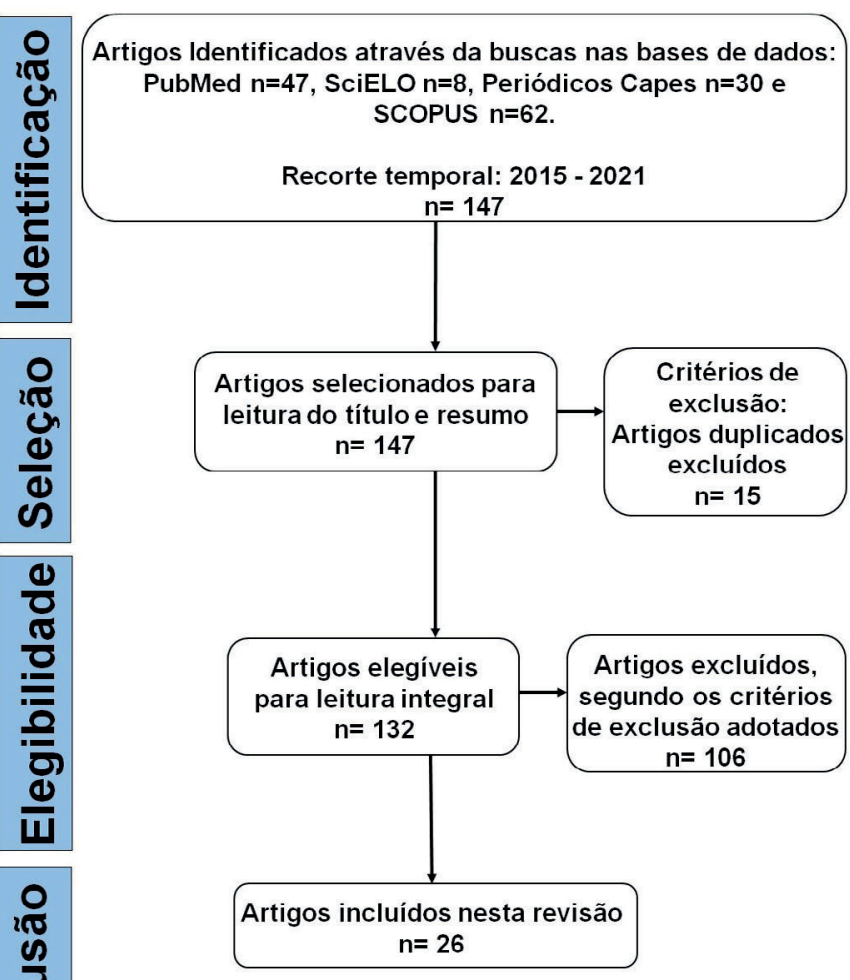

Figura 1. Algoritmo representativo da metodologia da pesquisa, fluxograma baseado no modelo PRISMA.

Fonte: Elaborado pelos autores.

\section{Resultados e Discussão}

A DP é uma doença neurodegenerativa incapacitante caracterizada pela tétrade clássica de bradicinesia, tremor de repouso, rigidez plástica e distúrbios posturais. ${ }^{1,3,7}$

O tremor é considerado como o sintoma inicial da DP, e em $50 \%$ dos indivíduos inicia-se nas extremidades distais. Esse aspecto é percebido nos períodos de repouso, e diminui ou desaparece com o estabelecimento de algum movimento. ${ }^{8}$

A bradicinesia, diminuição progressiva da velocidade e/ ou da amplitude de movimento, ocasionada pela ausência de dopamina no estriado é o sintoma mais incapacitante da DP. ${ }^{8}$ Ela provoca uma lentidão e um prolongamento do movimento, que por consequência ocasiona dependência das atividades diárias. ${ }^{5,9}$

Indivíduos com DP apresentam anormalidades de postura e do equilíbrio, as quais culminam em alterações da marcha. ${ }^{8}$ A marcha torna-se em bloco, exibindo-se com passos curtos, rápidos e arrastados, sem participação dos movimentos braçais. ${ }^{5,9} \mathrm{~A}$ postura é caracteriza pela cabeça anteriorizada e pelo aumento da cifose torácica com uma flexão de joelhos. Como o reflexo postural está diminuído, as quedas tornam-se mais frequentes. ${ }^{8}$

Além dos sintomas motores, a DP também apresenta sintomas não motores (SNM), cada vez mais reconhecidos por impactar na qualidade de vida dos pacientes. ${ }^{1,6,8}$ Dentre os SNM destaca-se os sensitivos, disautonômicos, involuntários, psiquiátricos, entre outros. Em se tratando dos sintomas involuntários, destaca-se a mioclonia e a distonia. ${ }^{1}$ Os sintomas psiquiátricos se revelam por depressão, ansiedade, apatia, agitação, ilusões e distúrbios do sono. ${ }^{5}$

\section{Tratamento da doença de Parkinson}

\section{Tratamento não farmacológico}

O Guideline da National Institute for Health and Care Excellence de 2017 cita como manejo não farmacológico dos sintomas motores e não motores da DP a fisioterapia, a atividade física, a terapia ocupacional, a terapia de linguagem, a nutrição e a intervenção especializada. Apesar da musicoterapia não estar na lista, tem recebido uma posição de destaque no que diz respeito à melhora dos pacientes em condições neurológicas, como Parkinson. ${ }^{13}$

\section{Musicoterapia}

Desde a antiguidade a música vem sendo utilizada como um instrumento terapêutico.5 Apenas no século $X X$, a musicoterapia se organiza no âmbito de aprofundar-se nas pesquisas acerca dos efeitos terapêuticos e preventivos que a música provoca nos seres humanos, sendo uma abordagem promissora. ${ }^{9,11}$

A União Brasileira das Associações de Musicoterapia (UBAM) define a musicoterapia como sendo "um campo de conhecimento que estuda os efeitos da música e da utilização de experiências musicais, resultantes do encontro entre o/a musicoterapeuta e as pessoas assistidas. A prática da Musicoterapia objetiva favorecer o aumento das possibilidades de existir e agir, seja no trabalho individual, com grupos, nas comunidades, organizações, instituições de saúde e sociedade, nos âmbitos da promoção, prevenção, reabilitação da saúde e de transformação de contextos sociais e comunitários; evitando dessa forma, que haja danos ou diminuição dos processos de desenvolvimento do potencial das pessoas e/ ou comunidades".14

A musicoterapia pode ser aplicada tanto individualmente como em grupo. Em grupo, busca-se o convívio e a amizade, o que é capaz de amenizar os aspectos psicológicos negativos relacionados à incapacidade física. Estimula-se ainda a comu- 
nicação interpessoal, o afeto, o aprendizado, proporcionando uma melhor qualidade de vida ao doente. De forma individual, personaliza-se a terapêutica aos diferentes quadros patológicos do paciente, como deficiência mental, perturbação motor, afecções psicossomáticas. ${ }^{11}$

A musicoterapia pode ser categorizada em duas abordagens: ativa ou receptiva. Na modalidade ativa, os pacientes participam do canto, tocam instrumentos musicais, fazem análises líricas, dançam e compõem músicas. Na modalidade receptiva, os participantes ouvem músicas, mas não é necessário tocar ou cantar. É importante ressaltar que ambas as abordagens envolvem musicoterapeutas e uma seleção de músicas baseada na preferência do paciente, o que ajuda a responder à intervenção, expressando seus sentimentos, emoções ou memórias despertadas., ${ }^{911}$

Na prática, os pacientes são estimulados a realizarem exercícios de voz associados a movimentos da mímica facial,15 exercícios de canto; exercícios de ritmo, enfatizando-se passos largos, postura ereta e balanço dos membros superiores. ${ }^{11}$

\section{Estimulação Auditiva Rítmica (EAR)}

Na DP, a supressão da secreção dopaminérgica aos núcleos da base (NB) acarreta hipoatividade do núcleo da base medial e das vias motoras complementares, o que altera a regulação interna da amplitude de movimento e do tempo. ${ }^{16}$ Esse processo leva a perda da capacidade de manter estabilidade rítmica da marcha. ${ }^{17}$ Os pacientes com DP têm dificuldade semelhante com as sincronizações rítmicas motoras e com a adaptação às mudanças no decorrer da deambulação. ${ }^{8}$

Visto que os déficits motores na DP são fortemente associados à falta de temporização, a estimulação auditiva rítmica (EAR), como metrônomo, batida musical ou ritmo de palmas, é uma estratégia promissora para reabilitação da marcha. ${ }^{4,8,18}$ Os benefícios da EAR se explicam uma vez que os pacientes apresentam um grau mínimo de processamento temporal dos estímulos externos, que podem ser suficientes para apoiar o início do movimento e sua execução. ${ }^{2,16}$ Sugere-se que a EAR permita a interação do córtex auditivo com um ou mais dos três principais sistemas neurais que controlam o desempenho motor: o sistema corticostriatal, o sistema corticocerebelar ou sistema pré-motor primário e o sistema cortical sensóriomotor. ${ }^{6,19}$ Esse processo é esclarecido pela atividade residual do circuito NB-talamocortical que determina a competência de elaborar previsões temporais por meio do tempo interno. ${ }^{2,15}$

No paciente parkinsoniano, há interrupção no eixo NB área motora suplementar - córtex pré-motor, o que gera recrutamento do cerebelo, órgão essencial para formulação tempo implícito. ${ }^{6,8}$ Esse incremento fornece ao doente a possibilidade de utilizar pistas rítmicas para auxiliar nas escolhas baseadas no tempo, como quando o próximo passo deve ocorrer. ${ }^{15}$

Exposições duradouras e prolongadas à música podem ampliar a formação de neurotrofinas produzidas em momentos de grande stress. O resultado é o aumento da sobrevida dos neurônios, além de modificações de parâmetros de conectividade na denominada neuroplasticidade. ${ }^{5,9,12,20} \mathrm{~A}$ música consegue aumentar a liberação de dopamina, diminuindo a sintomatologia na DP.,21 É possível arquitetar o fluxo cerebral $^{9}$, impulsionar e coordenar as atividades cerebrais, como modulação nos ritmos cardíaco e respiratório, modulação nos pulsos elétricos do cérebro e regulação do eixo sono-vigília. ${ }^{9}$

A EAR é capaz de aperfeiçoar a marcha por meio da geração de uma harmonia entre os passos e batida. A partir da música, um estímulo externo, as áreas parietal lateral, as áreas pré-motoras e o tálamo são parcialmente mais envolvidas. ${ }^{17}$ Dessa forma, torna-se possível aumentar o estímulo muscular e a excitabilidade dos neurônios dos circuitos dos núcleos da base com débito de dopamina. ${ }^{8-9}$

Durante a realização da terapia com EAR os pacientes são instruídos a deambular enquanto combinam seus passos com a batida da música ou do metrônomo, o que ajuda a superar o congelamento motor típico da DP. ${ }^{17,19}$ A instabilidade postural associada a complicações motoras adversas, que é dificilmente contida pela terapia farmacológica, também pode ser amenizada pela EAR. ${ }^{6}$

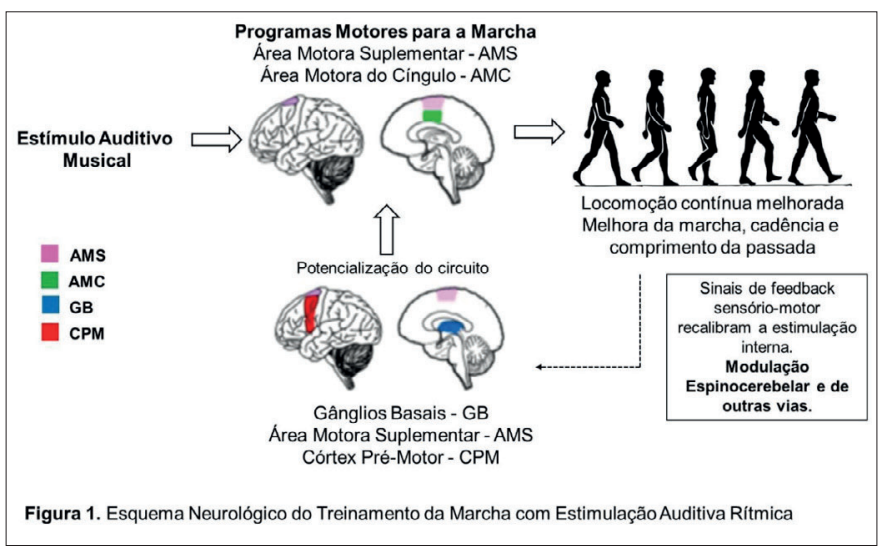

Figura 2. Esquema Neurológico do Treinamento da Marcha com Estimulação Auditiva Rítmica

Fonte: Adaptada de Dalla Bella et al., 2015.

Legenda: Devido ao tempo interno prejudicado, os programas motores realizados na AMS e AMC não são acessados facilmente sem o estímulo auditivo rítmico. A EAR é capaz de criar sinais de feedback sensoriais e motores que recalibram o sistema interno GBAMS-CPM, modulando vais como espinocerebelar, espinotalâmica, espinorreticular e espino-hipotalâmica. Dessa forma, o paciente adquire melhora da marcha, da locomoção contínua, da cadência e do comprimento do passo. 
Conclui-se que, a partir da EAR, ocorre aumento da excitabilidade dos neurônios motores espinais, por meio da via retículo espinal, diminuindo o tempo necessário para os músculos reagirem a um delimitado estímulo motor. ${ }^{9}$ Percebese o aperfeiçoamento da iniciação rítmica e do planejamento motor. ${ }^{2}$ Além disso, nota-se um ajuste na relação entre os fatores espaço-temporais da marcha e melhora na execução da dupla tarefa, isto é, escutar música (cognição) e deambular. ${ }^{8,9} \mathrm{O}$ esquema neurológico do treinamento da marcha com estimulação auditiva rítmica é ilustrado na figura 2.

\section{Efeitos e benefícios da musicoterapia}

A DP é uma doença crônica e progressiva, e ainda que a terapia farmacológica seja utilizada, não se observa a paralização do seu agravamento. ${ }^{8}$ Diante disso, recomenda-se a associação de algumas práticas não farmacológicas ao tratamento, como a musicoterapia. Esse recurso estimula o paciente a melhorar, manter ou restaurar um estado de bem-estar. ${ }^{9}$

A música tem uma grande influência nos processos cognitivos, sendo capaz de melhorar as funções cognitivas de atenção, percepção, linguagem, memória e raciocínio intelectual. ${ }^{6,9}$ Além disso, é notório a melhoria da marcha, no que concerne à velocidade, à cadência e à amplitude do passo. Os pacientes aprimoram o seu equilíbrio e diminuem a quantidade e o risco de quedas..$^{8,9}$

No decurso do tratamento auxiliar com a musicoterapia, pacientes aperfeiçoam a dicção e a expressão facial, sendo provocada por técnica vocal com movimentos de boca, língua e músculos da face. ${ }^{12,15}$ Exercícios rítmicos trouxeram benefícios para a coordenação motora limitada pelo enrijecimento muscular. ${ }^{6}$ Testemunhou-se aumento da autoestima, alegria e sociabilidade. A musicoterapia também contribui para uma melhor qualidade de vida, sociabilidade e redução nos níveis da depressão.,11 O quadro 1 sintetiza os principais benefícios da musicoterapia no tratamento adjuvante da doença de Parkinson.

\section{Quadro 1. Principais Benefícios da Musicoterapia no trata- mento auxiliar da doença de Parkinson}

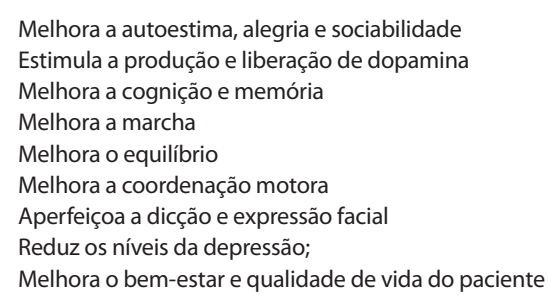

Fonte: MURGIA et al., 2018 (8); BELLA, 2018 (5); BRAUNLICH et al., 2018 (6); HARRISON, MCNEELY, EARHART, 2017 (17); ELLIS et al., 2015 (7); KHAN etal., 2015 (11);SCHIAVIO, ALTENMULLER, 2015 (22).

\section{Musicoterapia neurológica (MTN) no processo de} neurorreabilitação de doenças neurodegenerativas

A neurorreabilitação é um processo de tratamento que envolve profissionais de várias especialidades voltadas à recuperação parcial ou total de pessoas portadoras de algum tipo de deficiência neurológica física, sensorial e/ou cognitiva, definitivas ou temporárias. ${ }^{9}$ Este processo engloba o paciente, sua família e os especialistas responsáveis pelo atendimento. ${ }^{21} \mathrm{~A}$ equipe de especialistas deve discutir todas as dificuldades apresentadas pelo paciente, decidindo sobre o que é possível de ser alcançado através de um programa de tratamento. ${ }^{10,21}$ Os objetivos do programa de tratamento são definidos e acordados, posteriormente, são acompanhados para análise do progresso do tratamento. ${ }^{10}$

A musicoterapia é uma modalidade de neurorreabilitação que envolve profissionais especialistas em saúde mental com o objetivo de aumentar a plasticidade sináptica cerebral, o que supre as necessidades físicas, mentais, sociais e cognitivas dos parkinsonianos. ${ }^{9,11}$ Define-se musicoterapia neurológica (MTN) como aplicação terapêutica da música para estimular mudanças nas áreas cognitivas, motoras e de linguagem após doença neurológica. ${ }^{9}$ A musicoterapia neurológica abrange quatro pilares: a reabilitação guiada pela neurociência que engloba a terapêutica baseada em conceitos de estudos clínicos; o aprendizado motor rítmico; os modelos de plasticidade cerebral, pelos quais a música é capaz de estabelecer parâmetros da cadeia neural através da modulação temporal da entrada sensória; por fim, os padrões musicais, que funcionam como modelos de facilitação e padronização e que facilitam a melhora do movimento motor, da fala e das funções cognitivas. ${ }^{6,9,10,15}$

Três técnicas senso-motoras são tratadas na musicoterapia neurológica: EAR, que desenvolve e aprimora a trabalho motor rítmico psicológico; técnica do desempenho terapêutico com instrumentos musicais (TIMP), facilitando os movimentos funcionais; e Incremento Sensorial Padronizado (PSE), que sistematiza padrões de movimentos no tempo e espaço, facilitando atividades cotidianas. ${ }^{9-11}$ Existe uma potente conexão entre a marcha, o tempo interno inato e a percepção rítmica dos indivíduos, o que leva ao estudo dos benefícios de se aplicar a música como terapia na DP. Sabe-se que o tempo musical percebido pelos seres humanos compreende o intervalo de 40-300 bpm, sendo o intervalo de 120-130 o preferido. Esse ritmo confortável, utilizado como EARé capaz de adequar os parâmetros espaço-temporais da marcha. ${ }^{19}$

\section{Política Nacional de Práticas Integrativas de}

Complementares (PNPIC) no Sistema Único de Saúde (SUS)

Em 2006, quando foi criada a Política Nacional de Práticas Integrativas e Complementares (PNPIC), eram ofertados apenas cinco procedimentos. Após 10 anos, em 2017, foram incorporadas 
14 atividades, chegando as 19 práticas disponíveis atualmente à população, e dentre elas está a musicoterapia. Pacientes do Sistema Único de Saúde (SUS) serão beneficiados com 10 novas Práticas Integrativas e Complementares (PICS). Os tratamentos utilizam recursos terapêuticos, baseados em conhecimentos tradicionais, voltados para prevenir diversas doenças, como depressão e hipertensão. Com as novas atividades, ao todo, o SUS passa a ofertar 29 procedimentos à população. ${ }^{23}$

A política traz diretrizes gerais para a incorporação das práticas nos serviços e compete ao gestor municipal elaborar normas para inserção da PNPIC na rede municipal de saúde, contribuindo assim para a consolidação de um modelo de atenção humanizada e centrada na integralidade do indivíduo, o que fortalece os princípios fundamentais dos SUS.

A música é um potente estímulo multimodal que transmite informação visual, auditiva e motora para o cérebro, o qual tem uma rede específica para seu processamento, caracterizada por regiões fronto-tempo-parietais. Essa ativação é útil no tratamento da DP por meio da reabilitação e da estimulação de conexões neuronais alteradas. ${ }^{6}$ Alguns estudos científicos demonstrando a eficácia da musicoterapia no tratamento auxiliar da doença de Parkinson são apresentados, de forma sintetizada, na Tabela 1.

Tabela 1. Estudos científicos demonstrando a eficácia da musicoterapia no tratamento auxiliar da doença de Parkinson

\begin{tabular}{|c|c|c|c|}
\hline AUTORIA & $\begin{array}{c}\text { CARACTERÍSTICAS } \\
\text { DO ESTUDO }\end{array}$ & $\begin{array}{c}\text { MODELO DE } \\
\text { INTERVENÇÃO }\end{array}$ & RESULTADOS \\
\hline MURGIA et al., 2018 (8). & $\begin{array}{l}\text { Estudo clínico rando- } \\
\text { mizado; } N=32 \text { partici- } \\
\text { pantes DP; }\end{array}$ & $\begin{array}{l}\text { Duração de } 5 \text { sema- } \\
\text { nas, com } 2 \text { sessões } \\
\text { de } 45 \text { minutos em } \\
\text { cada uma, sendo } 20 \\
\text { dedicados ao treina- } \\
\text { mento com estimu- } \\
\text { lação auditiva rítmi- } \\
\text { ca (EAR) ecológica } \\
\text { ou artificial. Além } \\
\text { disso, foram convi- } \\
\text { dados a realizar o } \\
\text { mesmo protocolo } \\
\text { de EAR pelo menos } \\
3 \text { vezes/semana. A } \\
\text { avaliação foi feita } \\
\text { de forma individual. } \\
\text { Posteriormente, } \\
\text { realizaram os exer- } \\
\text { cícios diariamente } \\
\text { por } 12 \text { semanas } \\
\text { em suas casas, sem } \\
\text { supervisão. }\end{array}$ & $\begin{array}{l}\text { Programas de rea- } \\
\text { bilitação integrados } \\
\text { com a EAR são efi- } \\
\text { cazes, melhorando } \\
\text { a velocidade da } \\
\text { marcha, cadência, } \\
\text { comprimento e } \\
\text { largura do passo e } \\
\text { duração do ciclo da } \\
\text { marcha. }\end{array}$ \\
\hline
\end{tabular}

\begin{tabular}{|c|c|c|c|}
\hline $\begin{array}{l}\text { BRAULINCH et al, } \\
2018 \text { (6). }\end{array}$ & $\begin{array}{l}\text { Caso-Controle; } \\
\mathrm{N}=15 \text { pacientes DP } \\
\text { e } \mathrm{N}=14 \text { Indivíduos } \\
\text { Controles da mesma } \\
\text { idade. }\end{array}$ & $\begin{array}{l}\text { Os participantes } \\
\text { realizaram quatro } \\
\text { tarefas com ou sem } \\
\text { Estimulação Auditi- } \\
\text { va Rítmica (EAR) em } \\
\text { duas velocidades } \\
\text { diferentes, as quais } \\
\text { foram avaliadas } \\
\text { por meio da Resso- } \\
\text { nância Magnética } \\
\text { Funcional. } \\
\text { Imagens anatômicas } \\
\text { do cérebro dos } \\
\text { participantes foram } \\
\text { adquiridas no equi- } \\
\text { pamento de Resso- } \\
\text { nância Magnética } \\
\text { durante a execução } \\
\text { funcional das tare- } \\
\text { fas comportamen- } \\
\text { tais. Um software } \\
\text { foi utilizado para } \\
\text { a análise das } \\
\text { imagens cerebrais } \\
\text { correlacionando- } \\
\text {-as com as redes } \\
\text { de conectividade } \\
\text { funcional e os dados } \\
\text { do comportamento } \\
\text { motor rítmico. }\end{array}$ & $\begin{array}{l}\text { Os indivíduos com } \\
\text { DP apresentaram } \\
\text { uma conectividade } \\
\text { mais forte entre o } \\
\text { sistema auditivo, } \\
\text { controle executivo } \\
\text { e as redes motoras } \\
\text { (cerebelares), } \\
\text { compensando um } \\
\text { comprometimento } \\
\text { do sistema motor. }\end{array}$ \\
\hline READY et al., 2018 (4). & $\begin{array}{l}\text { Estudo clínico rando- } \\
\text { mizado } \\
\mathrm{N}=84 \text { indivíduos adul- } \\
\text { tos jovens }\end{array}$ & $\begin{array}{l}\text { A linha de base da } \\
\text { marcha dos pacien- } \\
\text { tes foi medida, os } \\
\text { quais selecionaram } \\
8 \text { músicas para o } \\
\text { teste de marcha. } \\
\text { Posteriormente, os } \\
\text { pacientes comple- } \\
\text { taram } 10 \text { ensaios } \\
\text { de marcha alea- } \\
\text { toriamente e por } \\
\text { último responderam } \\
\text { um questionário } \\
\text { relacionado a sua } \\
\text { história com a } \\
\text { dança e música e } \\
\text { um subteste para } \\
\text { medir a capacidade } \\
\text { de percepção da } \\
\text { batida. }\end{array}$ & $\begin{array}{l}\text { Foram analisados } \\
\text { comprimento da } \\
\text { passada, cadência, } \\
\text { velocidade e tempo } \\
\text { de suporte de mem- } \\
\text { bro duplo. A EAR } \\
\text { tem capacidade de } \\
\text { aumentar a estabi- } \\
\text { lidade da marcha, } \\
\text { quando combinada } \\
\text { com a percepção da } \\
\text { batida da música. }\end{array}$ \\
\hline DOTOV et al., 2017 (18). & $\begin{array}{l}\text { Caso-controle; } \mathrm{N}=19 \\
\text { pacientes com } \mathrm{DP} \text { e } \\
\mathrm{N}=19 \text { indivíduos } \\
\text { saudáveis. }\end{array}$ & $\begin{array}{l}\text { Foram realizadas } 18 \\
\text { sessões em } 3 \text { en- } \\
\text { saios de } 30 \text { minutos, } \\
\text { em que os pacientes } \\
\text { percorreram } 3,6 \\
\text { metros, durante } \\
2 \text { dias. Usou-se } \\
\text { fones de ouvido } \\
\text { via um sistema de } \\
\text { monitoramento de } \\
\text { som sem fio como } \\
\text { forma de transmis- } \\
\text { são da sugestão } \\
\text { auditiva. A taxa do } \\
\text { estímulo auditivo } \\
\text { foi aumentada em } \\
10 \% \text {. }\end{array}$ & $\begin{array}{l}\text { A partir da EAR, } \\
\text { evidenciou-se } \\
\text { sincronização dos } \\
\text { passos, aumento } \\
\text { no comprimento da } \\
\text { marcha e aumento } \\
\text { na velocidade da } \\
\text { marcha. }\end{array}$ \\
\hline
\end{tabular}




\begin{tabular}{|c|c|c|c|}
\hline $\begin{array}{l}\text { TOLLESON et al., } \\
2015 \text { (3). }\end{array}$ & $\begin{array}{l}\text { Estudo clínico rando- } \\
\text { mizado } \\
\mathrm{N}=14 \text { pacientes com } \\
\mathrm{DP} \text { e FOG (sintomas } \\
\text { de congelamento da } \\
\text { marcha); } \mathrm{N}=20 \text { com } \\
\text { DP sem FOG; } \mathrm{N}=19 \\
\text { Indivíduos saudáveis. }\end{array}$ & $\begin{array}{l}\text { Participantes } \\
\text { foram submetidos } \\
\text { a tarefas auditivas } \\
\text { rítmicas sincroni- } \\
\text { zadas analisadas } \\
\text { de acordo com o } \\
\text { paradigma de tem- } \\
\text { porização motora. }\end{array}$ & $\begin{array}{l}\text { Houve melhora } \\
\text { no tempo de sin- } \\
\text { cronização motor, } \\
\text { aprimorando a } \\
\text { marcha. }\end{array}$ \\
\hline
\end{tabular}

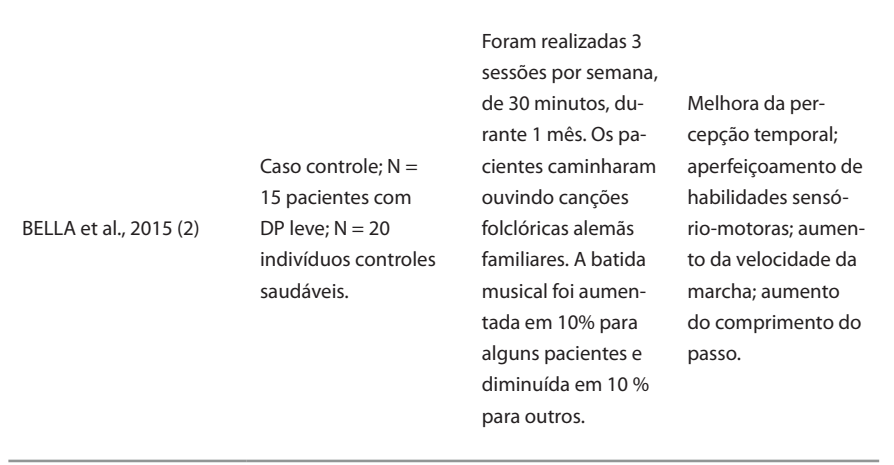

\begin{tabular}{|c|c|c|c|}
\hline BELLA et al., 2017 (24). & $\begin{array}{l}\text { Caso controle; } \mathrm{N}=14 \\
\text { pacientes com } \mathrm{DP} ; \\
\mathrm{N}=20 \text { indivíduos } \\
\text { controles saudáveis. }\end{array}$ & $\begin{array}{l}\text { Foram realizadas } 3 \\
\text { sessões por semana } \\
\text { durante } 4 \text { semanas. } \\
\text { Em } 7 \text { pacientes, } \\
\text { a frequência da } \\
\text { batida musical foi } \\
\text { aumentada em } \\
10 \% \text {. Nos outros } 7 \\
\text { pacientes, a frequ- } \\
\text { ência foi diminuída } \\
\text { em } 10 \% \text {. }\end{array}$ & $\begin{array}{l}\text { Utilizando-se a } \\
\text { técnica da EAR, } \\
\text { evidenciou-se que } \\
\text { os pacientes conse- } \\
\text { guiram uma melhor } \\
\text { sincronização dos } \\
\text { passos, um aumen- } \\
\text { to da velocidade da } \\
\text { marcha e um au- } \\
\text { mento significativo } \\
\text { do comprimento do } \\
\text { passo. }\end{array}$ \\
\hline
\end{tabular}

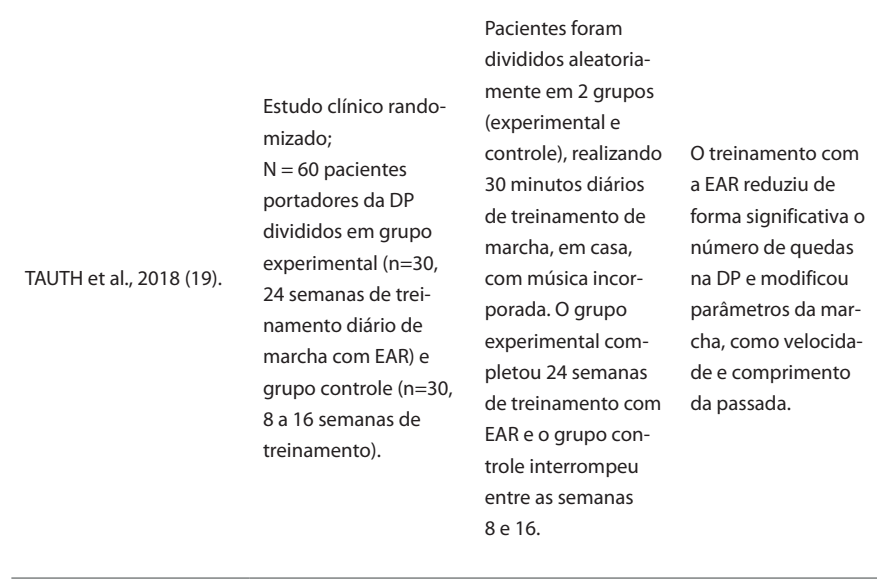

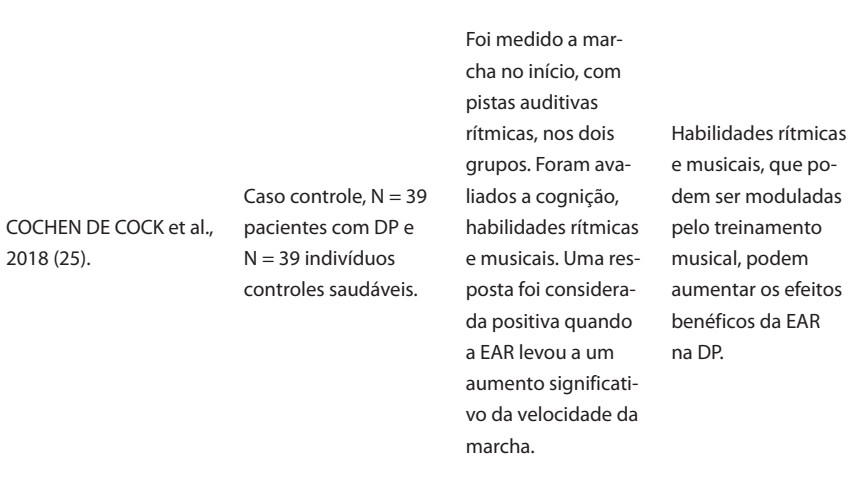

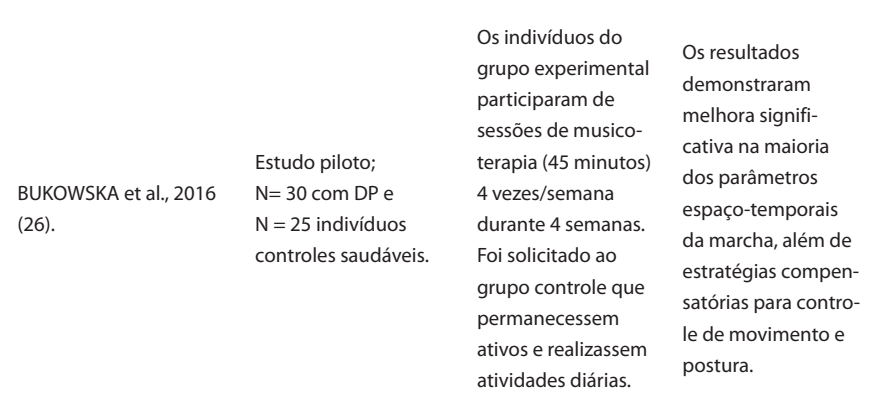

A terapia baseada na música apresenta excelentes resultados no tratamento de doenças que alteram a capacidade física, cognitiva e/ou subjetiva dos pacientes. Na tabela 1, destacamos dez estudos em que a musicoterapia apresentou resultados eficazes no tratamento auxiliar de pacientes com DP. De maneira geral, a terapêutica resultou na diminuição das exigências de equilíbrio postural, bem como no aumento do comprimento do passo do paciente e no aumento da velocidade da marcha, o que levou a uma maior independência funcional, acarretando desfechos positivos na progressão da doença.

A musicoterapia tem proporcionado melhor qualidade de vida aos pacientes, aliviando os sintomas motores e não motores ao estimular a produção dopaminérgica. ${ }^{8,9}$ À vista disso, o Sistema Único de Saúde do Brasil (SUS), em 2017, incluiu a musicoterapia no rol de procedimentos da Política Nacional de Práticas Integrativas e complementares.

Estudar a musicoterapia e seus efeitos benéficos, dessa forma, ampara os indivíduos com DP em várias competências. Há aperfeiçoamento das funções físicas e mentais atingidas, dado que a música propicia um sincronismo no sistema nervoso central, estimulando e coordenando as atividades do cérebro. Ocorre, também, minimização dos sintomas depressivos e de ansiedade advindos a partir da descoberta da doença, o indivíduo conseguirá lidar melhor com o seu sofrimento e com a sua insegurança.

\section{Conclusão}

A musicoterapia, portanto, tem se tornado uma ferramenta com grande potencial para o tratamento auxiliar da doença de Parkinson. Esse instrumento tem proporcionado melhor qualidade de vida aos pacientes, aliviando os sintomas motores e não motores ao estimular a produção dopaminérgica. Atualmente, a musicoterapia faz parte do rol de procedimentos terapêuticos da Política Nacional de Práticas Integrativas e complementares.

Esta terapia facilita o sequenciamento motor para geração de movimentos, o que favorece o engajamento do paciente, 
na realização de atividades, e, consequentemente, potencializa ganhos funcionais. Ainda, a técnica da EAR, uma ferramenta da musicoterapia, promove benefícios ao auxiliar no processo de temporização. O paciente, além de se sentir bem ao escutar uma música que o relaxe, tem a possibilidade de aumentar as atividades do complexo espaço-temporal, além de prolongar a vida dos neurônios.

Ademais, estudos foram realizados com pequeno universo amostral, intervenções curtas, avaliando apenas efeitos em curto prazo, além da maioria analisar pacientes avaliados com DP de leve a moderada. Assim, para explorar melhor o tema, são necessários mais ensaios clínicos randomizados utilizando-se a musicoterapeuta, maior universo amostral e tempo de intervenção, avaliando resultados a longo prazo e pacientes com DP grave.

\section{Referências}

1. Trost W, Leh F, Houvenaghel JF, Choppin S, Drapier S, Sauleau $\mathrm{P}$, et al. Subthalamic deep brain stimulation influences complex emotional musical experience in Parkinson's disease. Neuropsychologia. 2018; 117:278-286

2. Bella SD, Benoit CE, Farrugia N, Schwartze M, Kotz SA. Effects of musically cued gait training in Parkinson's disease: beyond a motor benefit. New York Academy of Sciences. 2015; 1337:77-85.

3. Tolleson CM, Dobolyi D, Roman OC, Kanoff K, Barton S, Wylie SA, et al. Dysrhythmia of time movements in Parkinson's disease and freezing of gait. Brain Research. 2015; 1624:222-231.

4. Ready EA, McGarry LM, Rinchon C, Holmes JD, Grahn JA. Beat perception ability and instructions to synchronize influence gait when walking to music-based auditory cues. Gait \& Posture. 2018; 68:555-561.

5. Bella SD. Music and moviment: Towards a translational approach. Neurophysiologie Clinique/ Clinical Neurophysiology. 2018; 48:377-386.

6. Braunlich K, Seger CA, Jentink KG, Buard I, Kluger BM, Thaut MH. Rythmic auditory cues shape neural network recruiment in Parkinson's disease during repetitive motor behavior. European Journal of Neuroscience. 2018; 49(6):849-858.

7. Ellis RJ, Ng YS, Zhu S, Tan DM, Anderson B, Schlaug G, et al. A validate Smartphone-Based Assessment of Gait and Gait Variability in Parkinson's Disease. Plos One. 2015; 10. Doi: 10.1371/journal.pone.0141694.

8. Murgia M, Pili R, Corona F, Sors F, Agostini TA, Bernardis P, et al. The Use of Footstep Sounds as Rhythmic Auditory Stimulaton for Gait Rehabilitation in Parkinson's Disease:
A Randomized Controlled Trial. Frontiers in Neurology. 2018; 9. Doi: 10.3389/fneur.2018.00348.

9. Moumdjian L, Sarkamo T, Leone C, Leman M, Feys P. Effectiveness of music-based interventions on motricity or cognitive functioning in neurological populations: A systematic review. European Journal of Physical and Rehabilitation Medicine. 2016; 53(3):466-482.

10. Robb SL, Hanson-Abromeit D, May L, Hernandez-Ruiz E, Allison M, Beloat A, et al. Reporting quality of music intervention research in healthcare: A systematic review. 2018; 38:24-41.

11. Khan WU, Yap IAMO, O'Neill D, Moss H. Perceptions of music therapy for older people among healthcare professionals. Med Humanit. 2015; 42:52-56.

12. Vik MBD, Skeie GO, Vikane E, Specht K. Effects of music production on cortical plasticity whitin cognitive rehabilitation on patients with mild traumatic brain injury. Brain Injury. 2018; 32(5):634-643.

13. National Institute for Health and Care Excellence NICE GUIDELINE. Parkinson's disease in adults. 2018. Disponível em: <https://www.nice.org.uk/terms-andconditions\#notice-ofrights $>$.

14. União Brasileira das Associações de Musicoterapia. Definição Brasileira de Musicoterapia. 2018. [acesso em 15 jul 2019]. Disponível em: <http://ubammusicoterapia.com.br/definicao-brasileira-demusicoterapia/>

15. Kotz SA, Gunter T. Can rythmic auditory cuing remediate language-related defecits in Parkinson's disease? New York Academy of sciences. 2015; 1337:62-68.

16. Harris R, Leenders K, De Jong BM. Speech dysprosody bust no music "disprosody" in Parkinson's disease. Brain \& Language. 2016; 163:1-9.

17. Harrison EC, Mcneely ME, Earthart GM. The feasibility of singing to improve gait in Parkinson disease. Gait \& Posture. 2017; 53:224-229.

18. Dotov DG, Bayard S, Cochen de Cock S, Geny C, Driss V, Garrigue $G$, atal. Biologically-variable rhythmic auditory cues are superior to isochronous cues in fostering natural gait variability in Parkinson's disease. Gait \& Posture. 2017; 51:64-69.

19. Thaut MH, Rice RR, Braun Janzen T, Hurt-Thaut CP, Mclntosh GC. Rythmic auditory stimulation for reduction of falls in Parkinson's disease: a randomized controlled study. Clinical Rehabilitation. 2018; 33(1):34-43.

20. Dauvergne C, Bégel V, Gény C, Puyjarinet F, Laffont I, Dalla Bella $S$. Home-based training of rythmic skills with a serious game in Parkinson's disease: Usability and acceptability. Physical and Rehabilitation Medicine. 2018; 61(6):380-385. 
21. Albani G, Veneziano G, Lunardon C, Vinci C, Daniele A, Cossa $F$, et al. Feasibility of home exercises to enhance the benefits of tango dancing in people with Parkinson's disease. Complementary Terapies in Medicine. 2019; 42:233-239.

22. Schiavio A, Altenmuller E. Exploring music-based rehabilitation for Parkinsonism through embodied cognitive Science. Frontiers in Neurology. 2015; 6. Doi: 10.3389/ fneur.2015.00217.

23. Valadares $C$. Ministério da Saúde inclui 10 novas práticas integrativas no SUS. 2018. [acesso em 30 jul 2019]. Disponível em: <http://www.saude.gov.br/noticias/ agencia-saude/42737-ministerio-da-saude-inclui10-novas-praticas-integrativas-no-sus>

24. Bella SD, Benoit CE, Farrugia N, Keller PE, Obrig H, Mainka $S$, et al. Gait improvement via rythmic stimulation in Parkinson's disease is linked to ryhtmic skills. Scientific Reports. 2017; 7. Doi: 10.1038/srep42005.

25. Cochen De CockV, Dotov DG, Ihalainen P, Bégel V, Galtier F, Lebrun, C, et al. Rhytyhmic abilities and musical training in Parkinson's disease: do they help? Nature Partner Journals. 2018. Doi: 10.1038/s41531-018-0043-7.

26. Bukowska AA, Krezalek P, Mirek E, Bujas P, Marchewka A. Neurologic Music Therapy Training for Mobility and Stability Rehabilitation with Parkinson's Disease - A Pilot Study. Frontiers in Human Neuroscience. 2016; 9. Doi: 10.3389/fnhum.2015.00710.

27. Souza MT, Silva MD, Carvalho R. Revisão integrativa: o que é e como fazer. Einstein. 2009;8(1):102-6.

28. Moher D, Liberati A, Tetzlaff J, Altman DG, Altman D, Antes $G$, et al. Preferred reporting items for systematic reviews and meta-analyses: The PRISMA statement. PLoS Med. 2009;6(7):e1000097. 